\title{
Research Article \\ Electrospinning of Cyclodextrin Nanofibers: The Effect of Process Parameters
}

\author{
Fuat Topuz $\mathbb{D}^{1}$ and Tamer Uyar $\mathbb{D}^{1,2}$ \\ ${ }^{1}$ Institute of Materials Science and Nanotechnology and UNAM-National Nanotechnology Research Center, Bilkent University, \\ Ankara 06800, Turkey \\ ${ }^{2}$ Department of Fiber Science and Apparel Design, College of Human Ecology, Cornell University, Ithaca, NY 14853, USA
}

Correspondence should be addressed to Fuat Topuz; topuzf@gmail.com and Tamer Uyar; tu46@cornell.edu

Received 24 April 2020; Accepted 23 May 2020; Published 15 June 2020

Academic Editor: Mohammad Rahimi-Gorji

Copyright ( 92020 Fuat Topuz and Tamer Uyar. This is an open access article distributed under the Creative Commons Attribution License, which permits unrestricted use, distribution, and reproduction in any medium, provided the original work is properly cited.

\begin{abstract}
Cyclodextrin (CD) nanofibers have recently emerged as high-performance materials owing to their large surface area-to-volume ratio, along with the presence of high active $\mathrm{CD}$ content for their applications in drug delivery and water treatment. Even though there are several studies on the polymer-free electrospinning of $\mathrm{CD}$ molecules of different types, the effects of electrospinning process parameters on the morphology and diameter of the resultant fibers have not addressed yet. In this study, the influence of electrospinning process variables on the morphology and diameter of the resultant CD nanofibers is systematically studied using two different solvent systems, i.e., water and N, N-dimethylformamide (DMF). On adjusting the electrospinning process parameters (i.e., electrical field, flow rate, tip-to-collector distance (TCD), and needle diameter), uniform CD nanofibers could be produced from aqueous and DMF solutions. Generally, the electrospinning of thicker fibers was observed by increasing the applied voltage and flow rate due to higher mass flow. Increasing TCD boosted the fiber diameter. Likewise, the use of needles with larger diameters resulted in the electrospinning of thicker fibers from DMF solutions, which might be attributed to higher viscosity due to reduced shear rate.
\end{abstract}

\section{Introduction}

Electrospinning is a fiber spinning technology that relies on the exposure of a viscoelastic solution to a high-voltage electrostatic field to produce a single charged jet and its deposition on a grounded collector in the form of nonwoven material with nanofibers from micrometer down to nanometer diameters (Figure 1(a)) [1]. In the first stage of the electrospinning process, a spherical droplet of a viscous solution turns into a conical-shape (i.e., so-called Taylor-cone) (Figures 1(b)-1(d)) and, then, ejects in an electrically charged continuous jet towards a collector plate. At the same time, the solvent is released from the fiber matrix until a solidified fiber is formed on the grounded plate. Throughout the process, several parameters are involved in the jet formation, drastically affecting the morphology and diameter of the resultant fibers (Figure 1(e)). In this regard, Haider et al. compiled a comprehensive review on the effects of electrospinning parameters on the morphology and diameter of the resultant fibers [2]. In this regard, both solution properties (e.g., viscosity, conductivity, and surface tension) and electrospinning process parameters, such as electrical field, flow rate, tip-tocollector distance (TCD), and needle diameter, affect the fiber properties, particularly the morphology, diameter, and size distribution.

Many studies reported the influence of process parameters on the electrospinning of polymeric systems. In this context, Okutan et al. described the influence of electrospinning process variables on the morphology and diameter of gelatin fibers [3]. An increase in the fiber diameter was observed with an electrical field rise, and bead-free smooth gelatin fibers could be produced. Theron et al. studied the electrospinning process parameters (i.e., the electrical field, solution flow rate, polymer concentration, molecular weight 


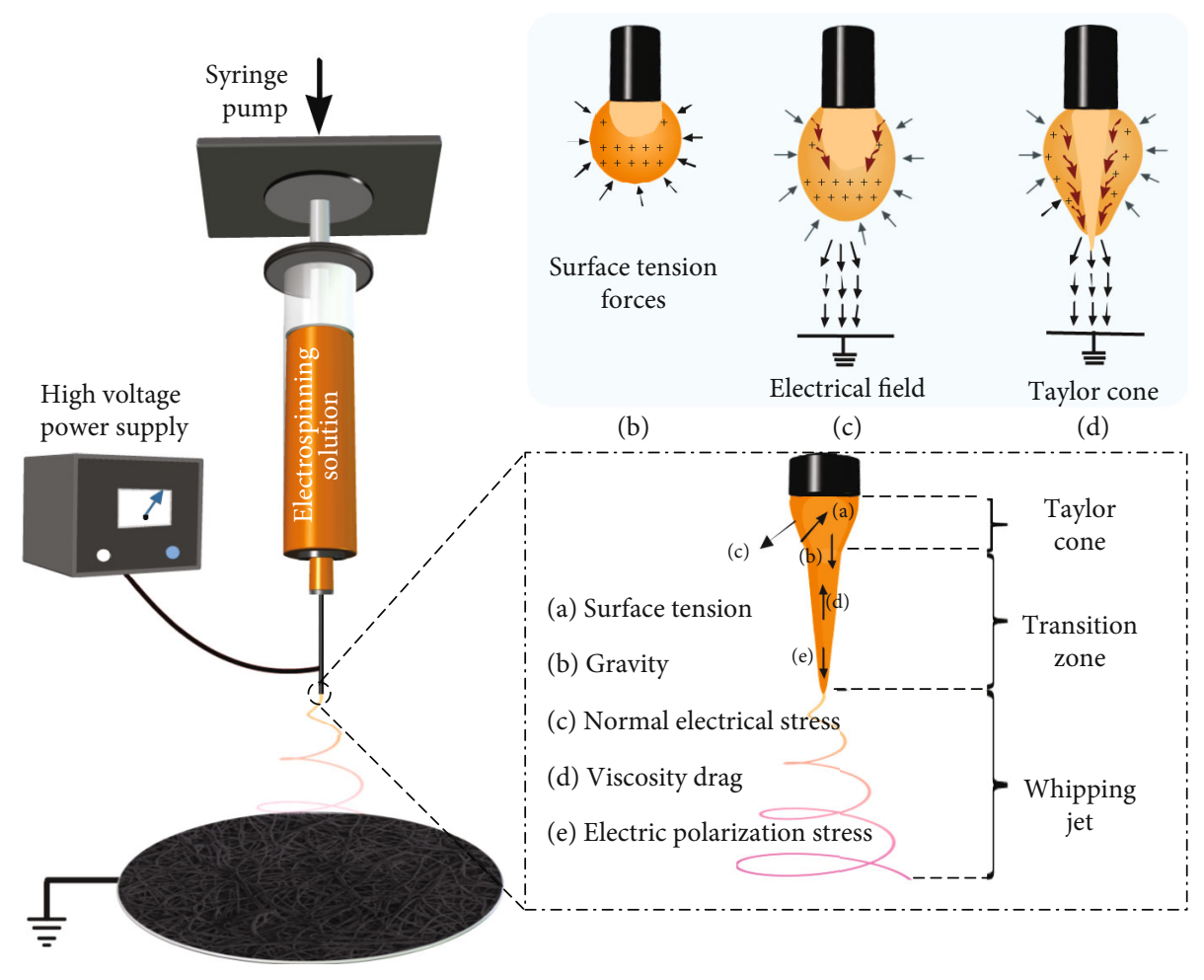

(a)

(e)

FIGURE 1: Cartoon illustration of a typical electrospinning setup with important parameters involving in the electrospinning process: (a) a general setup, (b-d) the formation of Taylor cone from the droplet, and (e) factors affecting the formation of the jet.

of polymer, and TCD) for several polymeric systems (i.e., poly(ethylene oxide) (PEO), poly(acrylic acid) (PAA), poly (vinyl alcohol) (PVA), polyurethane (PU), and polycaprolactone (PCL)) and observed important effects of the different process parameters on the electrical current and volume, as well as surface charge density in the polymer jet [4]. Amiraliyan et al. investigated the effect of electrospinning parameters on the morphology and diameter of Bombyx mori silk fibers [5]. They found that the fiber diameter was boosted with a concentration rise and observed the formation of ribbon-like fibers on increasing the electrospinning temperature. A systematic parameter study on the electrospinning of poly(L-lactic acid) (PLLA) fibers was reported for the production of uniform fibers [6]. The influence of process variables, including the electrical field and flow rate, on the electrospinning, was studied, and the authors observed the formation of nonuniform fibers with smaller diameters at very high voltages. Likewise, thicker fibers were obtained by increasing the flow rate.

The presence of surface charges on the fluid surface is crucial for the stretching of the jet during the electrospinning process (Figures 1(b)-1(e)). It ultimately determines the morphology and diameter of the resultant fibers. The electrical field can thus have a distinct effect on the fiber diameter since it is directly related to the number of charges applied to the solution [6]. In some studies, increasing the voltage boosted the mass flow drawn from the tip of the needle. Some studies reported the formation of thinner fibers on increasing the electrical field owing to the higher stretching of the electro-
TABle 1: Processing parameter description.

\begin{tabular}{|c|c|c|}
\hline Processing parameter & Units & Values \\
\hline Solvent & & Water, DMF \\
\hline HP- $\beta$-CD concentration & $\%(w / v)$ & $120,130,140$, and 180 \\
\hline Applied voltage & $\mathrm{kV}$ & $\begin{array}{c}7.5,10,12.5,15,17.5 \\
20, \text { and } 22\end{array}$ \\
\hline Flow rate & $\mathrm{mL} \cdot \mathrm{h}^{-1}$ & $0.1,0.25,0.75$, and 1 \\
\hline $\begin{array}{l}\text { Tip-to-collector } \\
\text { distance (TCD) }\end{array}$ & $\mathrm{cm}$ & $5,10,17.5,20$, and 25 \\
\hline Needle diameter & Gauge (G) & 18,20 , and 27 \\
\hline
\end{tabular}

spinning jet. However, some researchers observed an opposite trend, i.e., the formation of thicker fibers with a rise in the electrical field as a result of higher mass flow. In one example, Mazoochi et al. reported the formation of thicker fibers with a rise in the applied voltage, and above a critical voltage, the fiber diameter exhibited a reducing trend [7]. Likewise, the tip-to-collector distance (TCD) can also affect the properties of the electrospun fibers. Since the electrical field strength is inversely proportional to the distance between the metal collector and the tip of the needle, the TCD parameter might influence the fiber properties, particularly the evaporation of the solvent molecules and, thereby, the prevention of fused fibers. Many studies showed different effects of the TCD parameter on the morphology and diameter of the resultant fibers. In one example, at short TCD, fused fibers were generally formed, followed by individual 


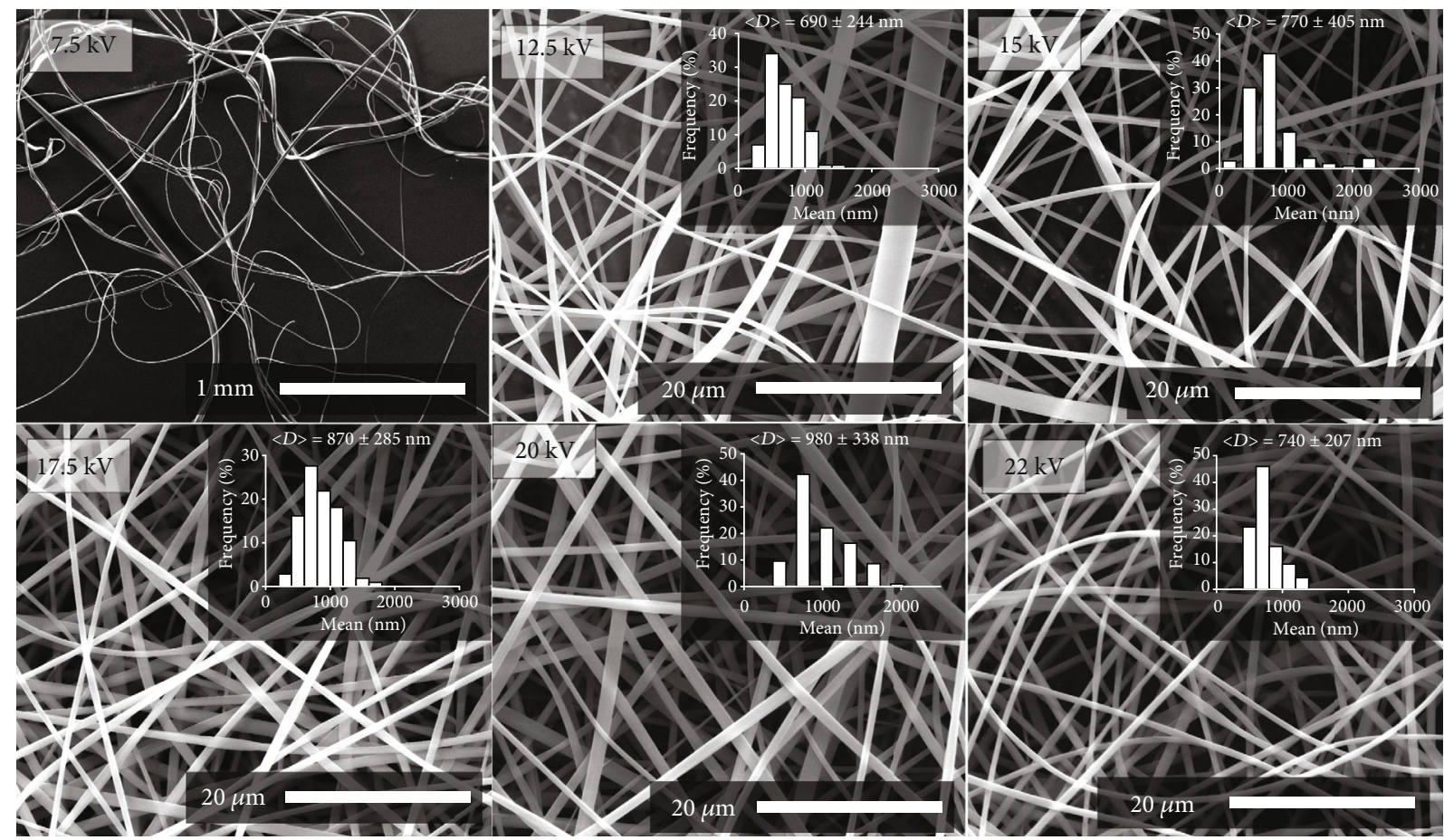

Figure 2: Scanning electron micrographs of HP- $\beta$-CD nanofibers produced from aqueous solutions at various voltages. The TCD and flow rate set were to $15 \mathrm{~cm}$ and $0.5 \mathrm{~mL} \cdot \mathrm{h}^{-1}$. Insets show the size distribution histograms of the respective fibers.

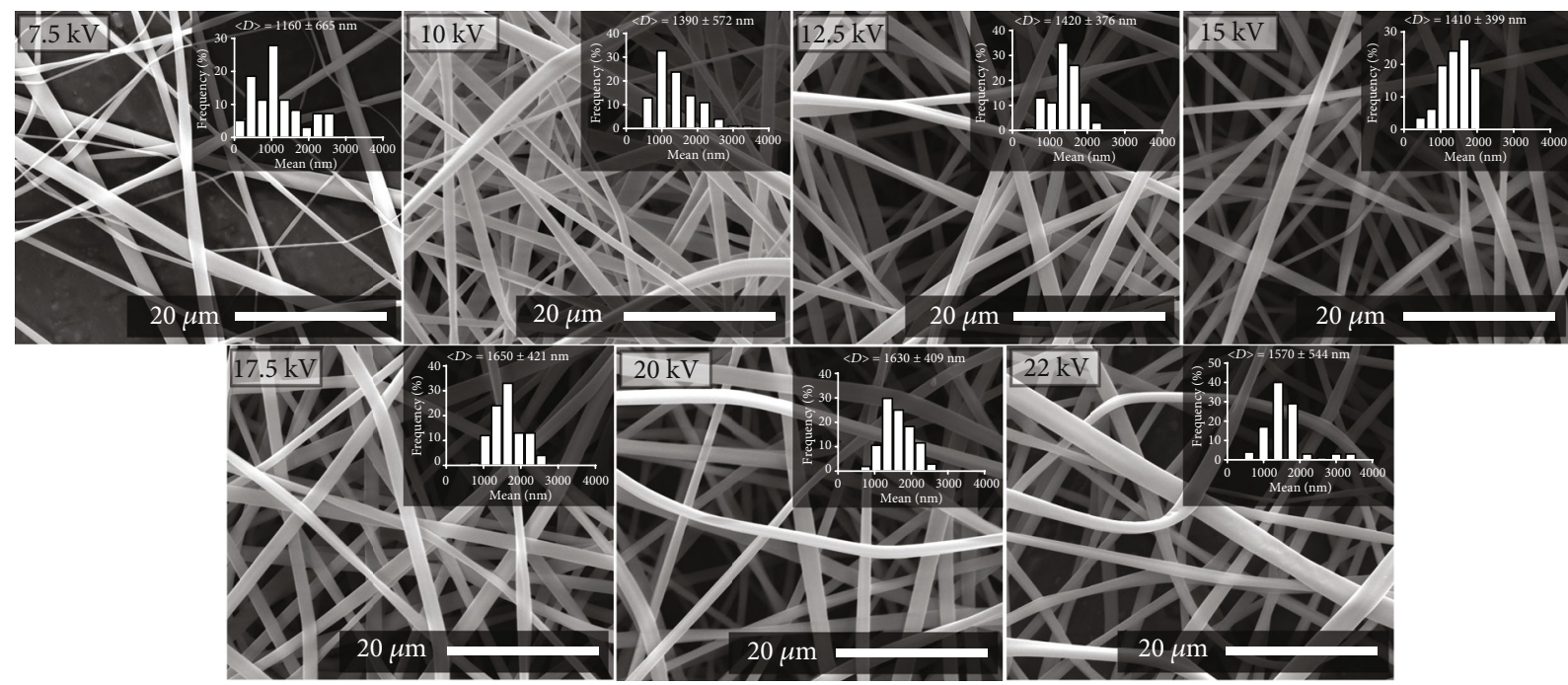

FIgURE 3: The influence of the electrical field on the resultant electrospun fibers produced from DMF solutions. The TCD and flow rate were, respectively, set to $15 \mathrm{~cm}$ and $0.5 \mathrm{~mL} \cdot \mathrm{h}^{-1}$. Insets show the size distribution histograms of the respective fibers.

fibers at typical TCD values. These fibers showed an increase in the fiber diameter at high TCD due to reduced electrostatic strength that led to lower jet stretching. Another critical electrospinning process parameter is the flow rate. It can significantly affect the diameter of the resultant fibers as the higher mass flow generally results in the formation of thicker fibers and nonuniform fiber morphology [8]. Likewise, the needle diameter can affect the initial jet diameter and the diameter of the resulting fibers. Unlike other parameters, the effect of needle diameter is rarely studied, and the formation of thicker fibers was observed with increasing the needle diameter [9]. Even though there are several studies that focuses on the effects of electrospinning process variables on the resultant fiber properties derived of polymeric systems, to the best of our knowledge, there has been no study focusing on the influence of electrospinning process variables for a polymer-free system, such as cyclodextrin (CD).

Cyclodextrin is a cyclic oligomer of glucopyranose with a truncated conical structure, whose interior has a partially hydrophobic character while the exterior is hydrophilic 


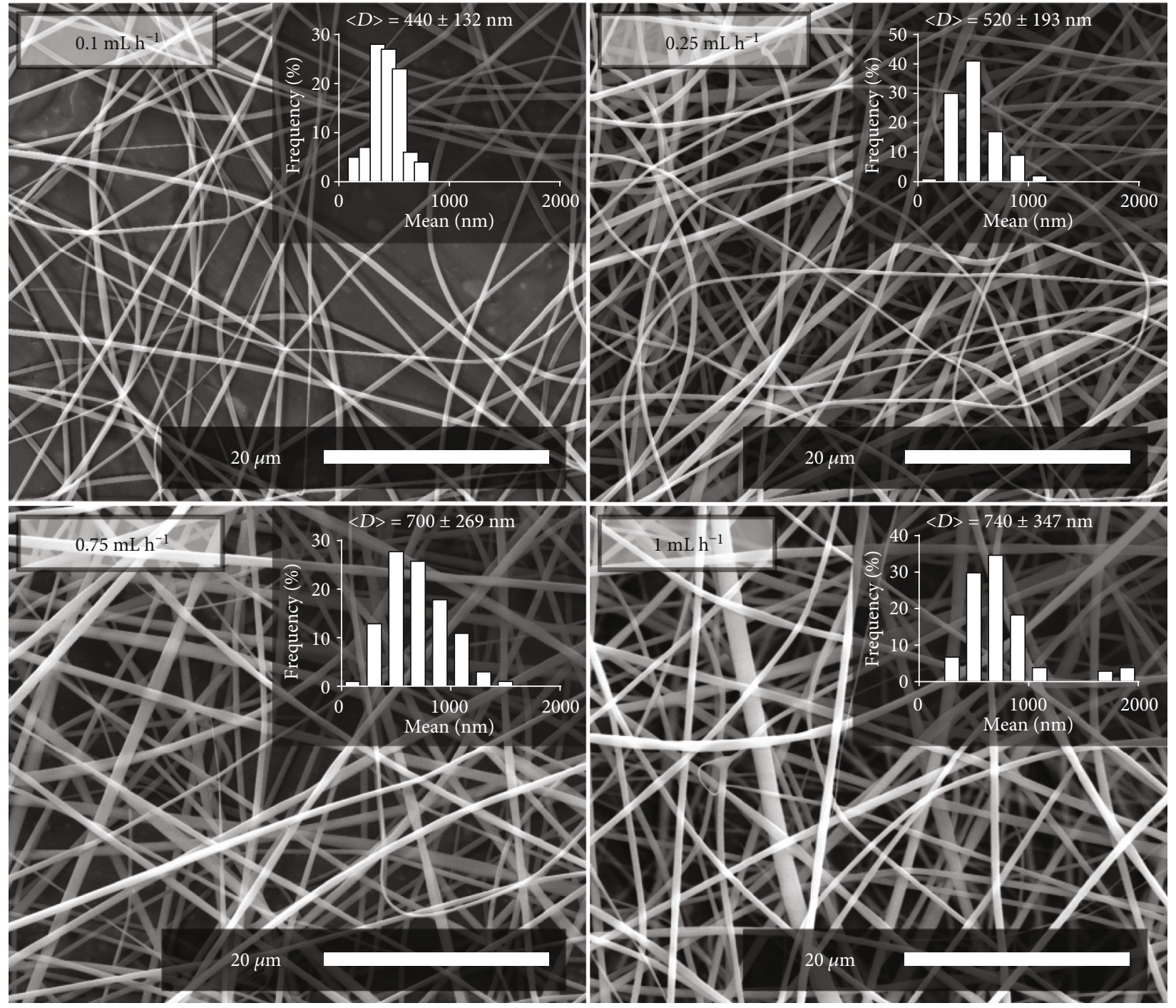

Figure 4: Scanning electron micrographs of HP- $\beta$-CD fibers produced from aqueous solutions at various flow rates. The applied voltage and TCD set to $17.5 \mathrm{kV}$ and $15 \mathrm{~cm}$. Insets show the size distribution histograms of the respective fibers.

owing to the presence of many hydroxyl groups [10]. They are biocompatible and edible biomolecules and have been exploited in numerous studies, including drug/gene delivery $[11,12]$, water treatment [13], textile [14], and food industry [15]. Among many different material forms of CD, electrospun CD nanofibers have sparked a considerable interest in the last decade owing to their potential use in a broad spectrum of applications. CD nanofibers could be produced without the need for any polymeric carrier by using different CD types, i.e., native [16], hydroxypropyl [17, 18], and methylated CDs [19]. The electrospinning of CD molecules in the absence of a polymeric component was attributed to the presence of hydrogen bonds $[20,21]$. Although the electrospinning of CD molecules of different types was previously reported, the influence of the electrospinning process parameters on the polymer-free CD fibers has remained as an unchallenged task to date. Thus, in this paper, we conducted a systematical study on the influence of electrospinning process parameters (i.e., electrical field, flow rate, tip-to-collector distance (TCD), and needle diameter) on the morphology and diameter of the $\mathrm{CD}$ fibers electrospun from their aqueous and organic (i.e., N, N-dimethylformamide) solutions.

\section{Experimental Section}

2.1. Materials. Hydroxypropyl- $\beta-\mathrm{CD}(\mathrm{HP}-\beta-\mathrm{CD}$, a molar substitution degree of 0.6-0.9) was kindly received as a gift from Wacker Chemie AG (Germany). The electrospinning solutions were prepared using high-purity water produced by a Millipore Milli-Q system or N, N-dimethylformamide (DMF, Sigma-Aldrich).

2.2. Electrospinning of $H P-\beta-C D$ Nanofibers. HP- $\beta-C D$ molecules were dissolved in water or DMF and transferred into a plastic disposable syringe having a metallic blunt-

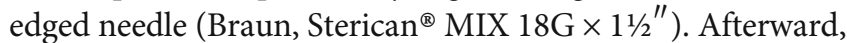
the syringe was horizontally placed on a KDS-101 model syringe pump, and a high voltage power supply (Matsusada Precision, AU Series) was connected. The nanofibers were collected on a metal collector covered with an aluminum foil. 

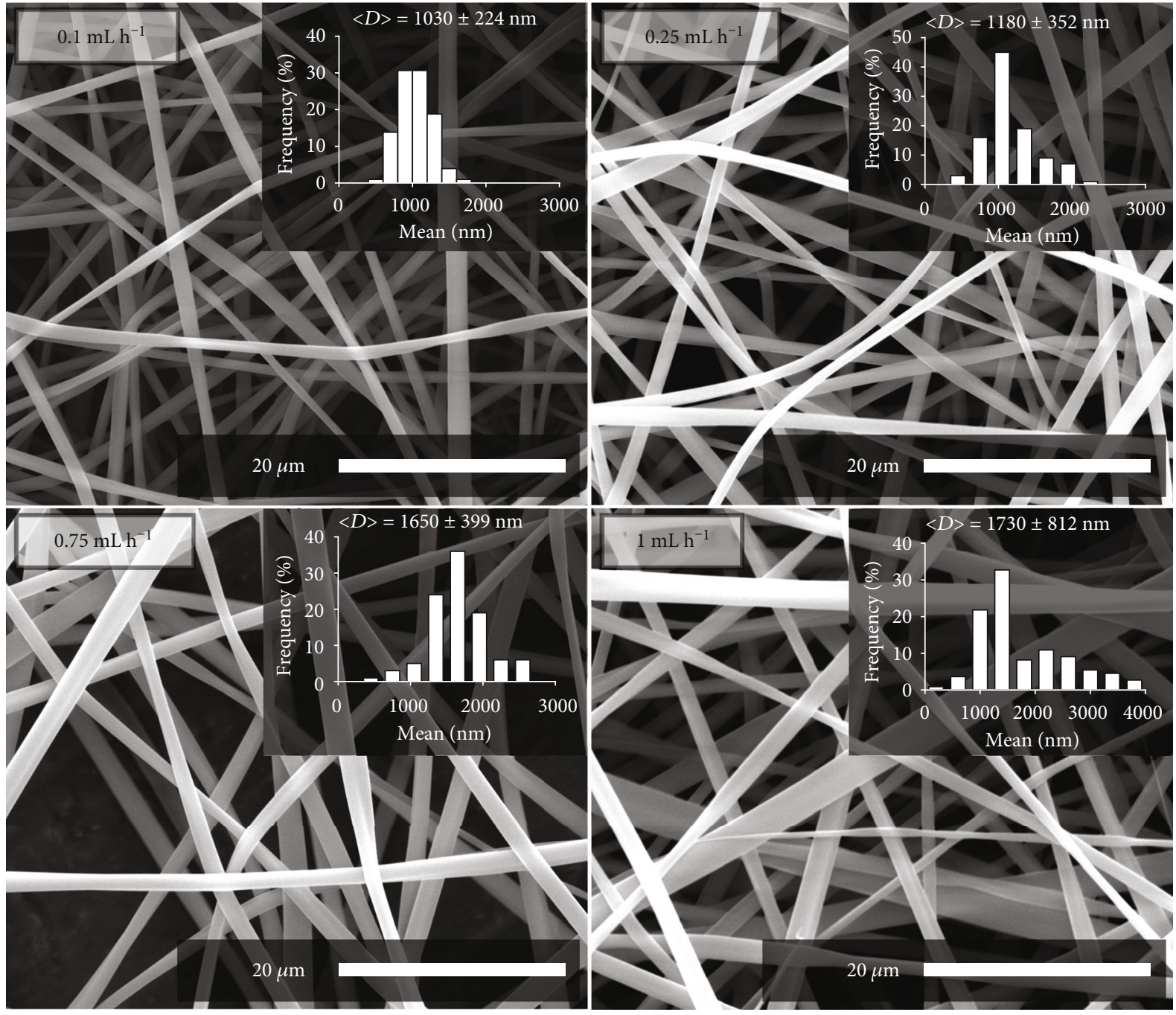

FIgURE 5: Scanning electron micrographs of HP- $\beta$-CD fibers produced from DMF solutions at various flow rates. The applied voltage and TCD were, respectively, set to $15 \mathrm{kV}$ and $15 \mathrm{~cm}$. Insets show the size distribution histograms of the respective fibers.

During the electrospinning, process variables, such as the electrical field, flow rate, tip-to-collector distance (TCD), and needle diameter, were systematically altered while keeping other electrospinning variables constant. During the electrospinning process, the relative humidity was $45 \pm 2 \%$ while temperature was maintained at $24 \pm 1^{\circ} \mathrm{C}$.

2.3. Scanning Electron Microscopy. The morphology of electrospun fibers was explored by scanning electron microscopy (SEM) (Quanta 200 FEG, FEI). Prior to the SEM analysis, the fiber specimens were coated with $5 \mathrm{~nm}$ Au with a PECS-682 sputter. The mean diameter of fibers was determined from the corresponding SEM images over $\sim 100$ fibers by ImageJ software (NIH, US National Institutes of Health).

\section{Results and Discussion}

First, the concentration of HP- $\beta$-CD was optimized to obtain bead-free nanofibers for each solvent system (water or DMF). For the electrospinning from DMF solutions, beaded-fibers were observed when the HP- $\beta-\mathrm{CD}$ concentration was 120 and $130 \%(w / v)$, while bead-free uniform nanofibers could be produced at the concentration of $140 \%(w / v)$ (Figure S1). Likewise, the electrospinning from aqueous $\mathrm{CD}$ solutions showed apparent changes depending on the HP- $\beta-C D$ concentration. Bead-free fibers could be produced when the HP- $\beta$-CD concentration was $180 \%(w / v)$. The texture of the fiber surface was smooth due to the gradual evaporation of solvent molecules during the electrospinning process. Unlike the electrospinning from DMF, the electrospinning of aqueous $\mathrm{CD}$ solutions led to fibers in a wide diameter distribution. Even though a higher concentration was used for the water-based electrospinning, the mean diameter of the resultant fibers was much lower than the fibers produced from DMF solutions. These concentrations are higher than the previously reported values, which are 120 and $160 \%(w / v)$ for DMF and aqueous solutions, respectively [17]. This increase can be attributed to the higher relative humidity: the relative humidity was $45 \%$ during the electrospinning of $\mathrm{CD}$ molecules, while the previously reported concentrations for the $\mathrm{CD}$ electrospinning were based on the relative humidity of $30 \%$ [17]. 

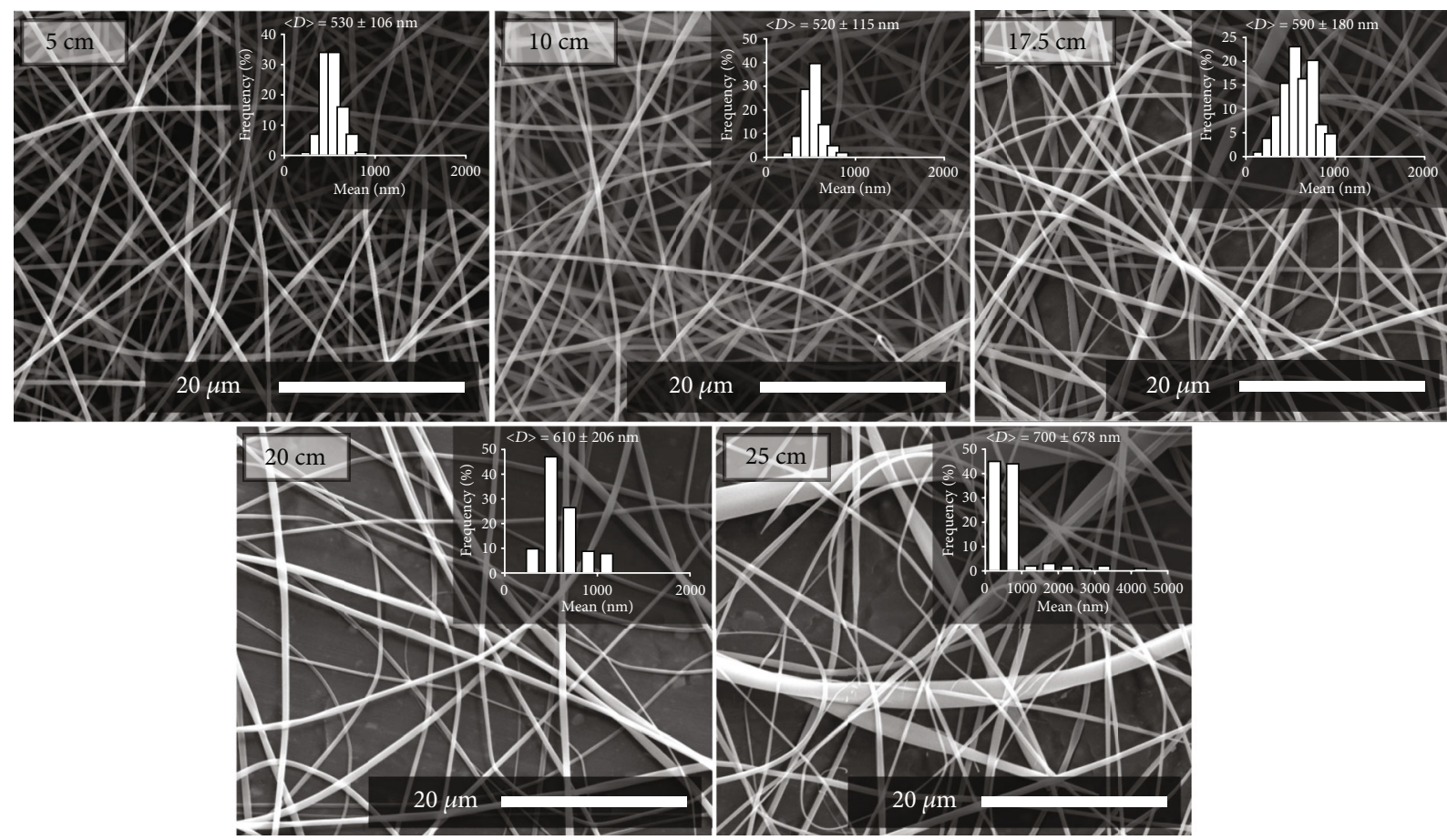

FIGURE 6: Scanning electron micrographs of HP- $\beta$-CD fibers produced from aqueous solutions at various TCDs. The applied voltage and flow rate were, respectively, set to $17.5 \mathrm{kV}$ and $0.25 \mathrm{~mL} \cdot \mathrm{h}^{-1}$. Insets show the size distribution histograms of the respective fibers.

3.1. Effect of Electrical Field. For the electrospinning of CD nanofibers, electrospinning process variables were systematically changed (see Table 1). CD fibers were produced using two different solvents: water and DMF. As a first parameter, the applied electrospinning voltage was systematically increased from 7.5 to $22 \mathrm{kV}$ while keeping other processing parameters constant (i.e., the TCD of $15 \mathrm{~cm}$ and the flow rate of $0.5 \mathrm{~mL} \cdot \mathrm{h}^{-1}$ ). Under an electrical field, the electrospinning droplet is deformed to a Taylor cone, then ejects to form fibers at a critical voltage. Figure 2 shows the SEM photos of the fibers electrospun from aqueous HP- $\beta$-CD solutions at various voltages. At all voltages, the formation of beadfree fibers was observed. However, increasing the electrical field boosted the fiber diameter: the mean diameter of the fibers produced at $12.5 \mathrm{kV}$ was $690 \pm 224 \mathrm{~nm}$ while it increased to $980 \pm 338 \mathrm{~nm}$ at the applied voltage of $20 \mathrm{kV}$. This increase can be attributed to the higher mass flow from increasing the electrical field. A further increase in the electrical field worsened the fiber morphology and resulted in nonuniform nanofibers. In this regard, a similar decrease in the fiber diameter at high voltages was observed for polyvinylpyrrolidone (PVP) [22] and poly(vinylidene fluoride) (PVDF) [23]. The electrospun nanofibers produced at the applied voltage of $17.5 \mathrm{kV}$ yielded the most uniform morphology for the applied voltages screened for the electrospinning of aqueous CD solutions.

Likewise, the influence of the electrical field on the electrospinning of HP- $\beta$-CD fibers from DMF solutions was also explored. Unlike the nanofibers produced from aqueous solutions, the electrospinning of CD from DMF solutions produces thicker fibers [17]. Unlike the electrospinning from aqueous solutions, the formation of thicker fibers even at lower $\mathrm{CD}$ concentrations can be attributed to the interactions between $\mathrm{CD}$ and DMF. Like CD fibers produced from aqueous solutions, a similar trend on the fiber diameter was observed on increasing the electrical field, and thicker fibers were observed with an applied voltage rise (Figure 3). The fibers produced at $7.5 \mathrm{kV}$ have a mean diameter of $1160 \pm$ $665 \mathrm{~nm}$, while it increased to $1570 \pm 544 \mathrm{~nm}$ with increasing the applied voltage to $22 \mathrm{kV}$. A higher voltage led to a higher mass flow and ultimately gave rise to the formation of thicker CD fibers. After a critical voltage, the thinner CD fibers were formed as observed for the electrospinning of aqueous $\mathrm{CD}$ solutions (Figures 2 and 3).

3.2. Effect of Flow Rate. As a next step, the flow rate was systematically increased while keeping the other parameters constant (i.e., applied voltage of $17.5 \mathrm{kV}$ and TCD of $15 \mathrm{~cm}$ ). It is known that the fiber diameter increases with the flow rate, and the formation of beaded-fibers were reported at high flow rates [8]. Increasing the flow rate of aqueous HP- $\beta$-CD solutions led to the formation of thicker fibers and gave rise to a broad size distribution: the fibers produced at the flow rate of $0.1 \mathrm{~mL} \cdot \mathrm{h}^{-1}$ has a mean diameter of $440 \pm 132 \mathrm{~nm}$ while the fibers produced at $1 \mathrm{~mL} \cdot \mathrm{h}^{-1}$ has a mean diameter of $740 \pm 347 \mathrm{~nm}$ (Figure 4).

A similar trend was observed for the fibers spun from DMF solutions: the mean fiber diameter increased with a flow rate rise (Figure 5). The electrospinning at the flow rate of $0.1 \mathrm{~mL} \cdot \mathrm{h}^{-1}$ led to fibers with a mean diameter of $1030 \pm$ $224 \mathrm{~nm}$, while the value increased to $1730 \pm 812 \mathrm{~nm}$ when increasing the flow rate by 10 -fold to $1 \mathrm{~mL} \cdot \mathrm{h}^{-1}$. This is a general trend for electrospinning, and thicker fibers are obtained with a higher flow rate as a result of increased mass flow. A 

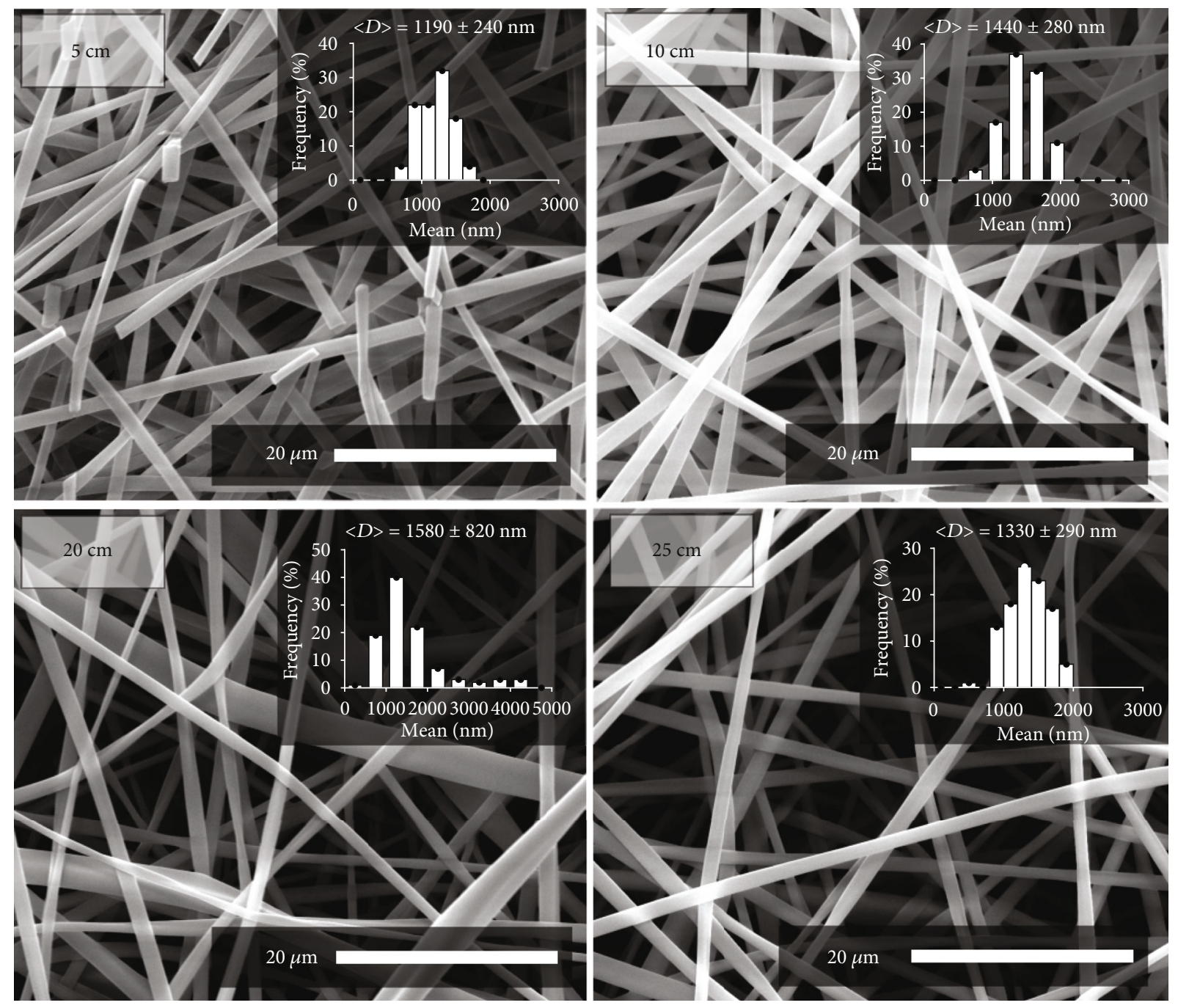

FIgURE 7: Scanning electron micrographs of HP- $\beta$-CD fibers produced from DMF solutions at various TCDs. The applied voltage and flow rate were, respectively, set to $15 \mathrm{kV}$ and $0.25 \mathrm{~mL} \cdot \mathrm{h}^{-1}$. Insets show the size distribution histograms of the respective fibers.

higher mass flow also led to fibers with a wide diameter distribution. In this regard, Milleret et al. studied the effect of the flow rate on the electrospinning of poly(lactic-co-glycolic acid) (PLGA) and observed the formation of thicker poly(lactic acid) fibers with a flow rate rise [24]. Likewise, Wang et al. reported the formation of thicker fibers of poly(lactic acid) with an increasing flow rate [25].

3.3. Effect of Tip-to-Collector Distance. The distance between the needle and collector can change the fiber properties, particularly texture and diameter, as it affects the electrical field strength between the collector and tip. Particularly, the TCD has a significant role in the deposition time, evaporation rate, and whipping $[2,26]$. Higher TCD may lead to the thinning of the electrospun fibers since there will be a greater stretching distance. However, after a certain distance, the fiber diameter might increase as a result of a weak electrical field. The effect of tip-to-collector distance (TCD) was also explored for the electrospinning of HP- $\beta$-CD from aqueous solutions (Figure 6). After a slight decrease in the fiber diameter with increasing TCD from 5 to $10 \mathrm{~cm}$, the formation of thicker fibers with a rise in TCD was apparent. The mean fiber diameter increased from $530 \pm 106 \mathrm{~nm}$ to $700 \pm 678$ $\mathrm{nm}$ with increasing TCD from 5 to $25 \mathrm{~cm}$. In addition to the thinner nanofibers, microfibers were also observed. Typically, the formation of thinner fibers is expected since increasing the TCD distance should lead to a mass loss. The occurrence of thicker fibers can be attributed to the weakening of the electrical field between the tip and collector, and thereby, the electrospinning yield drastically drops. The droplet gets larger, and when electrospinning occurs, thicker and nonuniform fibers are formed.

A similar trend was also observed for the fibers electrospun from DMF solutions. Thicker fibers were formed by increasing the flow rate (Figure 7). The mean diameter of the fibers spun at $5 \mathrm{~cm}$ increased from $1190 \pm 240$ to $1580 \pm 820 \mathrm{~nm}$ by increasing TCD from 5 to $20 \mathrm{~cm}$. Higher TCD also increased the diameter distribution range, so the fibers spun at higher TCDs were formed in a broader diameter range. On the other hand, the formation of thinner fibers was observed with a further rise in TCD to $25 \mathrm{~cm}$ due to the reduced electrical field. 

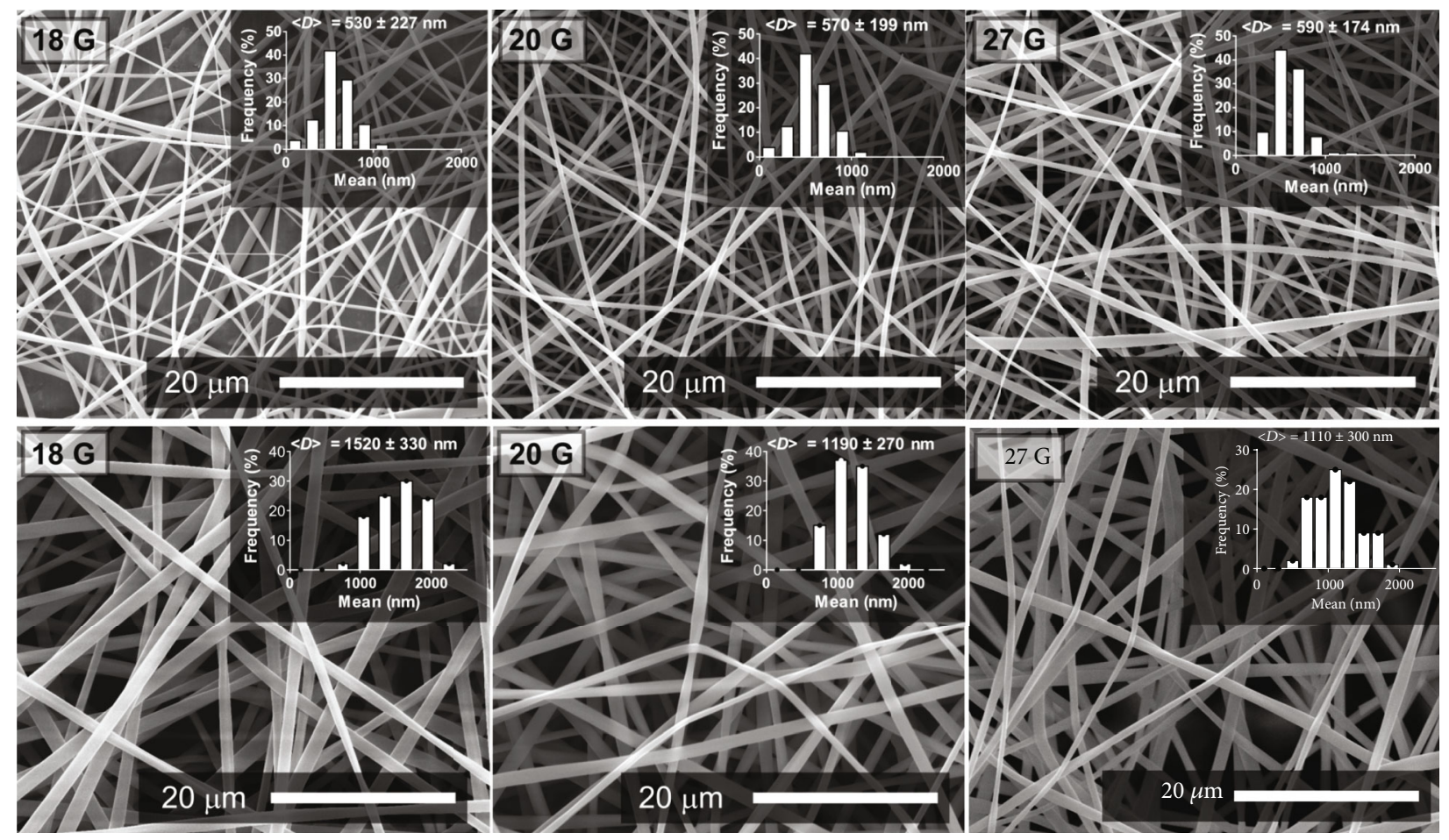

FIgURE 8: Scanning electron micrographs of HP- $\beta$-CD fibers produced from aqueous solutions (top panel) and DMF solutions (bottom panel) using different diameter needles. The applied voltage, flow rate, and TCD were, respectively, set to $17.5 \mathrm{kV}$ (for water-based electrospinning) or $15 \mathrm{kV}$ (for DMF-based electrospinning), $0.25 \mathrm{~mL} \cdot \mathrm{h}^{-1}$, and $10 \mathrm{~cm}$. Insets show the size distribution histograms of the respective fibers.

3.4. Effect of Needle Diameter. The influence of the needle diameter on the fiber morphology and diameter was also explored for HP- $\beta$-CD the electrospinning from aqueous and DMF solutions (Figure 8). For the CD fibers produced from aqueous solutions, the needle diameter slightly affected the fiber diameter: thicker fibers were formed when the needles with smaller diameters were used. The nanofibers produced from aqueous $\mathrm{CD}$ solutions using an $18 \mathrm{G}$ needle (inner diameter $=0.838 \mathrm{~mm}$ ) have a mean fiber diameter of $530 \pm 227 \mathrm{~nm}$ while it slightly increased to $590 \pm 174 \mathrm{~nm}$ with a $27 \mathrm{G}$ needle (inner diameter $=0.210 \mathrm{~mm}$ ). This increase in the fiber diameter can be attributed to the formation of a larger volume of electrospinning solution on the needle head, which resulted in thicker fibers. The influence of needle diameter on the electrospinning of HP- $\beta$-CD fibers from DMF solutions was also explored. The SEM images of the respective fibers were shown in Figure 8, where thinner nanofibers were formed with decreasing the needle diameter: for instance, the mean diameter of electrospun fibers produced from DMF solutions using $18 \mathrm{G}$ needle was $1520 \pm$ $330 \mathrm{~nm}$, while the internal diameter of the fibers prepared by $27 \mathrm{G}$ needle was $1110 \pm 300 \mathrm{~nm}$, demonstrating the formation of larger fibers when larger diameter needles were used. The formation of thicker fibers can be attributed to the larger amount of mass available for the electrospinning. The previous studies reported the formation of thicker fibers by increasing the needle diameter. In one example, the final jet radius was related to the initial jet radius and the formation of poly(lactide-co-glycolide) (PLAGA) fibers with mean

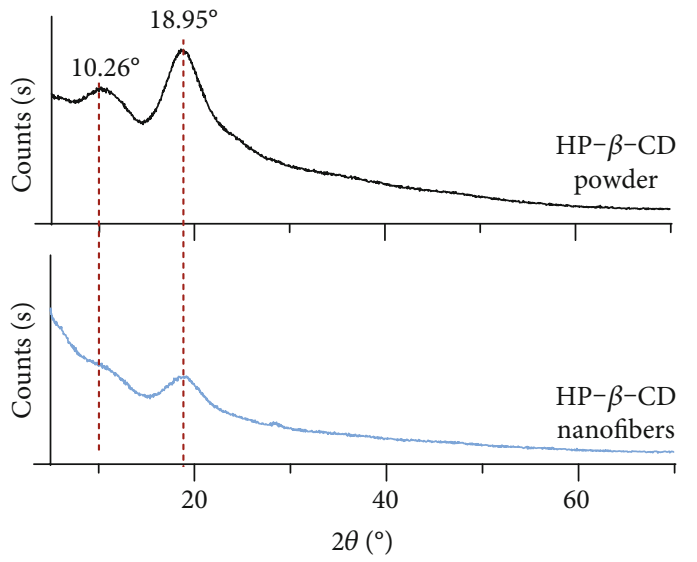

FIgURE 9: Wide-angle XRD patterns of the HP- $\beta$-CD powder and nanofibers.

diameters of 240, 150, and $125 \mathrm{~nm}$ was achieved for needles with radii of $0.059,0.042$, and $0.029 \mathrm{~cm}$, respectively, [9]. This might be attributed to the decrease in the shear rate, which leads to a higher viscosity, and as known, a higher viscosity leads to the formation of thicker fibers.

The structural analysis of the HP- $\beta$-CD nanofibers was explored through WAXS where broad amorphous peaks appeared at $10.26^{\circ}$ and $18.95^{\circ}$ with the respective $d$-spacing values of 8.61 and $4.67 \AA$ (Figure 9). Those peaks are lower than the powder form of HP- $\beta-C D$, demonstrating a decrease in the amorphous structure of the HP- $\beta$-CD after 


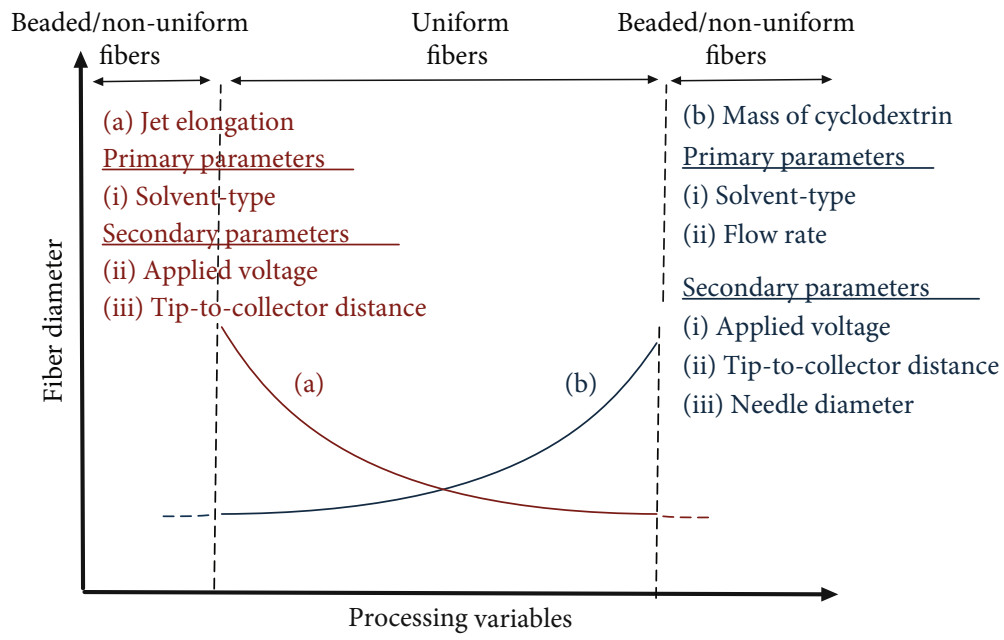

FIGURE 10: Processing map obtained based on the systematic variable study: (a) jet elongation (influenced by solvent type, applied voltage, and tip-to-collector distance), (b) mass of cyclodextrin (mainly affected by solvent-type and flow rate, while slightly affected by applied voltage, tip-to-collector distance, and needle diameter).

the electrospinning process. The effect of electrospinning on the molecular structure was also explored over FTIR analysis. Figure S2 shows the FTIR spectra of both powder and nanofibers of the HP- $\beta-\mathrm{CD}$ where lower intensity peaks were observed after the electrospinning without any change in the peak positions, demonstrating that the molecular structure was preserved after the electrospinning process.

The overall influence of electrospinning process variables on the diameter of the resultant fibers was illustrated in Figure 10. The diameter of the electrospun fibers showed significant alterations with variations in the process variables. The thinning of electrospun fibers was due to the jet elongation and observed for the electrospinning from aqueous solutions. The changes in applied voltage and tip-to-collector distance also led to the higher jet elongation and thereby fiber thinning. Regardless of the solvent type, the increase the process parameters (i.e., applied voltage, flow rate, tip-tocollector distance, and inner needle diameter) led to the formation of larger fibers for water and DMF-based electrospinning due to a higher $\mathrm{CD}$ mass transport from the needle (Figure 10).

\section{Conclusion}

The effects of electrospinning process variables on the morphology and diameter of $\mathrm{CD}$ nanofibers produced from aqueous and DMF solutions were systematically studied. With the tuning of the electrospinning process variables, the CD fibers were produced in the diameter range of $440-$ $980 \mathrm{~nm}$ for aqueous solutions and $1030-1730 \mathrm{~nm}$ for DMF solutions. The electrospinning of CD nanofibers from DMF solutions could be produced at a lower concentration than aqueous solutions. Generally, the increase in the electrospinning process variables led to thicker fibers. The electrospinning from $\mathrm{CD}$ solutions at increasing electrical fields resulted in the formation of thicker fibers. Regardless of the solvent type used, similar effects of electrospinning parameters were observed on the resultant nanofibers. Thicker fibers were produced by increasing the electrical field. Likewise, changes in other process parameters have led to size variations on $\mathrm{CD}$ fibers; i.e., higher TCD and flow rate induced thicker fibers. At very high voltages and TCD, the electrospinning of thinner fibers was observed due to reduced electrical field. Overall, the effects of process parameters on $\mathrm{CD}$ fibers were similar to those of polymeric ones and coincides well the reports of polymer electrospinning. Tuning the process variable led to the formation of uniform electrospun $\mathrm{CD}$ fibers for both water and DMF solutions.

\section{Data Availability}

The data used to support the findings of this study are included within the article.

\section{Conflicts of Interest}

There is no conflict of interest.

\section{Supplementary Materials}

SEM photos of the HP- $\beta$-CD fibers produced at various concentrations and FTIR spectra of the HP- $\beta$-CD powder and fibers are available in the Supplementary material file. (Supplementary Materials)

\section{References}

[1] Y. M. Shin, M. M. Hohman, M. P. Brenner, and G. C. Rutledge, "Electrospinning: a whipping fluid jet generates submicron polymer fibers," Applied Physics Letters, vol. 78, no. 8, pp. 1149-1151, 2001.

[2] A. Haider, S. Haider, and I.-K. Kang, "A comprehensive review summarizing the effect of electrospinning parameters and potential applications of nanofibers in biomedical and biotechnology," Arabian Journal of Chemistry, vol. 11, no. 8, pp. 1165-1188, 2018. 
[3] N. Okutan, P. Terzi, and F. Altay, "Affecting parameters on electrospinning process and characterization of electrospun gelatin nanofibers," Food Hydrocolloids, vol. 39, pp. 19-26, 2014.

[4] S. A. Theron, E. Zussman, and A. L. Yarin, "Experimental investigation of the governing parameters in the electrospinning of polymer solutions," Polymer, vol. 45, no. 6, pp. 20172030, 2004.

[5] N. Amiraliyan, M. Nouri, and M. H. Kish, "Effects of some electrospinning parameters on morphology of natural silkbased nanofibers," Journal of Applied Polymer Science, vol. 113, no. 1, pp. 226-234, 2009.

[6] S. H. Tan, R. Inai, M. Kotaki, and S. Ramakrishna, "Systematic parameter study for ultra-fine fiber fabrication via electrospinning process," Polymer, vol. 46, no. 16, pp. 6128-6134, 2005.

[7] T. Mazoochi, M. Hamadanian, M. Ahmadi, and V. Jabbari, "Investigation on the morphological characteristics of nanofiberous membrane as electrospun in the different processing parameters," International Journal of Industrial Chemistry, vol. 3, no. 1, p. 2, 2012.

[8] S. Zargham, S. Bazgir, A. Tavakoli, A. S. Rashidi, and R. Damerchely, "The effect of flow rate on morphology and deposition area of electrospun nylon 6 nanofiber," Journal of Engineered Fibers and Fabrics, vol. 7, no. 4, 2018.

[9] D. S. Katti, K. W. Robinson, F. K. Ko, and C. T. Laurencin, "Bioresorbable nanofiber-based systems for wound healing and drug delivery: optimization of fabrication parameters," Journal of Biomedical Materials Research, vol. 70B, no. 2, pp. 286-296, 2004.

[10] G. Crini, "Review: a history of cyclodextrins," Chemical Reviews, vol. 114, no. 21, pp. 10940-10975, 2014.

[11] T. Loftsson, P. Jarho, M. Másson, and T. Järvinen, "Cyclodextrins in drug delivery," Expert Opinion on Drug Delivery, vol. 2, no. 2, pp. 335-351, 2005.

[12] F. Topuz and T. Uyar, "Electrospinning of cyclodextrin functional nanofibers for drug delivery applications," Pharmaceutics, vol. 11, no. 1, p. 6, 2019.

[13] A. Celebioglu, F. Topuz, Z. I. Yildiz, and T. Uyar, "Efficient removal of polycyclic aromatic hydrocarbons and heavy metals from water by electrospun nanofibrous polycyclodextrin membranes," ACS Omega, vol. 4, no. 4, pp. 7850-7860, 2019.

[14] M. Mirjalili and S. Zohoori, "Review for application of electrospinning and electrospun nanofibers technology in textile industry," Journal of Nanostructure in Chemistry, vol. 6, no. 3, pp. 207-213, 2016.

[15] M. Noruzi, "Electrospun nanofibres in agriculture and the food industry: a review," Journal of the Science of Food and Agriculture, vol. 96, no. 14, pp. 4663-4678, 2016.

[16] T. Kida, S.-i. Sato, H. Yoshida, A. Teragaki, and M. Akashi, "1, 1,1, 3, 3,3-Hexafluoro-2-propanol (HFIP) as a novel and effective solvent to facilely prepare cyclodextrin-assembled materials," Chemical Communications, vol. 50, no. 91, pp. 1424514248, 2014.

[17] A. Celebioglu and T. Uyar, "Electrospinning of nanofibers from non-polymeric systems: polymer-free nanofibers from cyclodextrin derivatives," Nanoscale, vol. 4, no. 2, pp. 621631, 2012.

[18] A. Celebioglu and T. Uyar, "Electrospinning of cyclodextrins: hydroxypropyl-alpha-cyclodextrin nanofibers," Journal of Materials Science, vol. 55, no. 1, pp. 404-420, 2020.
[19] A. Celebioglu and T. Uyar, "Cyclodextrin nanofibers by electrospinning," Chemical Communications, vol. 46, no. 37, pp. 6903-6905, 2010.

[20] J. L. Manasco, C. D. Saquing, C. Tang, and S. A. Khan, "Cyclodextrin fibers via polymer-free electrospinning," RSC Advances, vol. 2, no. 9, pp. 3778-3784, 2012.

[21] F. Topuz and T. Uyar, "Influence of hydrogen-bonding additives on electrospinning of cyclodextrin nanofibers," ACS Omega, vol. 3, no. 12, pp. 18311-18322, 2018.

[22] P. Korycka, A. Mirek, K. Kramek-Romanowska, M. Grzeczkowicz, and D. Lewińska, "Effect of electrospinning process variables on the size of polymer fibers and bead-onstring structures established with a 23 factorial design," Beilstein Journal of Nanotechnology, vol. 9, pp. 2466-2478, 2018.

[23] H. Shao, J. Fang, H. Wang, and T. Lin, "Effect of electrospinning parameters and polymer concentrations on mechanical-toelectrical energy conversion of randomly-oriented electrospun poly (vinylidene fluoride) nanofiber mats," RSC Advances, vol. 5, no. 19, pp. 14345-14350, 2015.

[24] V. Milleret, B. Simona, P. Neuenschwander, and H. Hall, "Tuning electrospinning parameters for production of 3Dfiberfleeces with increased porosity for soft tissue engineering applications," European Cells and Materials, vol. 21, pp. 286303, 2011.

[25] C. Wang, H.-S. Chien, K.-W. Yan et al., "Correlation between processing parameters and microstructure of electrospun poly( $D$,l-lactic acid) nanofibers," Polymer, vol. 50, no. 25, pp. 6100-6110, 2009.

[26] K. P. Matabola and R. M. Moutloali, "The influence of electrospinning parameters on the morphology and diameter of poly (vinyledene fluoride) nanofibers- effect of sodium chloride," Journal of Materials Science, vol. 48, no. 16, pp. 5475-5482, 2013. 